\title{
Sets of reflections defining twisted Bruhat orders
}

\author{
Tom Edgar
}

Received: 17 May 2006 / Accepted: 22 January 2007 /

Published online: 29 March 2007

(C) Springer Science+Business Media, LLC 2007

\begin{abstract}
Twisted Bruhat orders are certain partial orders on a Coxeter system $(W, S)$ associated to initial sections of reflection orders, which are certain subsets of the set of reflections $T$ of a Coxeter system. We determine which subsets of $T$ give rise to a partial order on $W$ in the same way.
\end{abstract}

\section{Introduction and preliminaries}

In a Coxeter system, $(W, S)$, reflection orders are certain total orders of the set of reflections $T$. Initial sections of these reflection orders, which are certain subsets of $T$, lead to partial orders (twisted Bruhat orders) on $W$ that are similar to Bruhat order. In fact, using the initial section $\emptyset \subseteq T$ we get the Bruhat order on $W$. In this paper, we determine all subsets of $T$ that give rise to partial orders on $W$ in the same manner. We see that subsets of $T$ that have this property are closely related to initial sections of reflection orders and are conjecturally the same.

I am grateful to Matthew Dyer for his suggestions regarding this paper as well as his guidance in making this paper more readable.

\section{Statement of main result}

We begin with the same setup as [3]. We let $(W, S)$ be a Coxeter system with $\ell: W \rightarrow \mathbb{N}$ the corresponding length function. Then, let $T=\cup_{w \in W} w S w^{-1}$ be the set of reflections of $W$, and regard $\mathcal{P}(T)$ as an Abelian group under symmetric difference. We define $N: W \rightarrow \mathcal{P}(T)$ by $N(w)=\{t \in T \mid \ell(t w)<\ell(w)\}$. By [2], $N$ can be characterized by $N(s)=\{s\}(\forall s \in S)$ and $N(x y)=N(x)+x N(y) x^{-1}(\forall x, y \in W)$.

T. Edgar $(\varangle)$

Department of Mathematics, University of Notre Dame, Notre Dame, IN 46556, USA

e-mail: tedgar@nd.edu 
This implies there is a $W$-action on $\mathcal{P}(T)$ given by $w \cdot A=N(w)+w A w^{-1}$ (where $w \in W$ and $A \subseteq T)$. For a reflection subgroup $W^{\prime}$ of $W$, we write $\chi\left(W^{\prime}\right)$ for the canonical set of generators for $W^{\prime}$, where $\chi\left(W^{\prime}\right)=\left\{t \in T \mid N(t) \cap W^{\prime}=\{t\}\right\}$. Due to [2], $N_{\left(W^{\prime}, \chi\left(W^{\prime}\right)\right)}(x)=N(x) \cap W^{\prime}$ for all reflection subgroups $W^{\prime}$ where $N_{\left(W^{\prime}, \chi\left(W^{\prime}\right)\right)}$ is the $N$ function for $\left(W^{\prime}, \chi\left(W^{\prime}\right)\right)$. Additionally, let $\Phi$ be the root system for $(W, S)$ with positive root system $\Phi^{+}$. For general reference regarding Coxeter groups, see [5].

Now, for any $A \subseteq T$, we can define a directed graph $\Omega_{(W, A)}$ with the vertex set of $\Omega_{(W, A)}$ equal to $W$. We define the edge set $E_{(W, A)}=\{(t w, w) \mid t \in w \cdot A\}$. Conjugating by $w$, we get the equivalent statement $E_{(W, A)}=\left\{(w t, w) \mid t \in N\left(w^{-1}\right)+A\right\}$ (see [3]). For $x \in W$, the map $w \mapsto w x$ defines an isomorphism $\Omega_{(W, A)} \cong \Omega_{(W, x \cdot A)}$. In addition, for $A \subseteq T$ we can define a length function $\ell_{A}: W \rightarrow \mathbb{Z}$ in the following way:

$$
\ell_{A}(v, w)=\ell\left(w v^{-1}\right)-2 \#\left[N\left(v w^{-1}\right) \cap v \cdot A\right] \in \mathbb{Z}
$$

and then set $\ell_{A}(w)=\ell_{A}(1, w)$. We can define a pre-order $\leq_{A}$ for any $A \subseteq T$ given by the following: $v \leq_{A} w$ if and only if there exist $t_{1}, \ldots, t_{n} \in T$ with $w=v t_{1} \ldots t_{n}$ such that $t_{i} \notin\left[N\left(\left(v t_{1} \ldots t_{i-1}\right)^{-1}\right)+A\right]$ for all $i=1, \ldots, n$.

Definition 2.1 Following [4], we call a total order $\prec$ on $T$ a reflection order if for any dihedral reflection subgroup $W^{\prime}$ of $W$ either $r \prec r s r \prec \cdots \prec s r s \prec s$ or $s \prec '$ srs $\prec^{\prime} \cdots \prec^{\prime} r s r \prec^{\prime} r$ where $\chi\left(W^{\prime}\right)=\{r, s\}$.

Remark 2.2 We can also define a reflection order in terms of the root system $\Phi$ associated to the Coxeter system. This definition can be found in [1].

Recall from [4] that an initial section of a reflection order is a subset $A \subseteq T$ such that there is a reflection order $\prec$ with the property that $a \prec b$ for all $a \in A$ and $b \in$ $T \backslash A$. It is shown in [3] that $\leq_{A}$ is a partial order of $W$ if $A$ is an initial section of a reflection order. Our main result, Theorem 2.3 below, describes all subsets $A$ of $T$ for which $\leq_{A}$ is a partial order.

Let $\mathbf{A}_{(W, S)}$ be the set of initial sections of reflection orders of $T$. Now, we define $\hat{\mathbf{A}}_{(W, S)}=\left\{A \subseteq T \mid A \cap W^{\prime} \in \mathbf{A}_{\left(W^{\prime}, \chi\left(W^{\prime}\right)\right)} \forall W^{\prime} \subseteq W\right.$ dihedral $\}$. It has been conjectured by Matthew Dyer that $\mathbf{A}=\hat{\mathbf{A}}$. We now come to the main result:

Theorem 2.3 Let $(W, S)$ be any Coxeter system. The following are equivalent:

1. $\Omega_{(W, A)}$ is acyclic.

2. $\leq_{A}$ is a partial order.

3. $\Omega_{(W, A)}$ has no cycle of length four.

4. $A \in \hat{\mathbf{A}}$.

5. $\ell_{A}(x t)<\ell_{A}(x)$ for all $x \in W$ and $t \in N\left(x^{-1}\right)+A$.

\section{Proof of main result}

In the following proofs, for any positive root $\alpha \in \Phi^{+}$, let $t_{\alpha} \in T$ be the corresponding reflection, and for any reflection $t \in T$, let $\alpha_{t} \in \Phi^{+}$be the corresponding positive 
root. To begin with we investigate the dihedral case. Suppose $(W, S)$ is dihedral, i.e. $S=\{r, s\}$. There is a bijection between subsets $A \subseteq T$ and subsets $\Psi \subset \Phi$ such that $\Psi \cup-\Psi=\Phi$ and $\Psi \cap-\Psi=\emptyset$ given by $A=A_{\Psi}=\left\{t_{\alpha} \mid \alpha \in \Psi \cap \Phi^{+}\right\}$. We note that $A_{-\Psi}=A_{\Psi}+T$.

Lemma 3.1 For any $A_{\Psi} \subseteq T, w \cdot A_{\Psi}=A_{w(\Psi)}$.

Proof It is enough to show that this is true for $r \in S$. Now we know that $r \cdot A_{\Psi}=$ $\{r\}+r A_{\Psi} r=\{r\}+\left\{r t_{\alpha} r \mid \alpha \in \Psi \cap \Phi^{+}\right\}=\{r\}+\left\{t_{r(\alpha)} \mid \alpha \in \Psi \cap \Phi^{+}\right\}=\{r\}+\left\{t_{\alpha} \mid \alpha \in\right.$ $\left.r(\Psi) \cap r\left(\Phi^{+}\right)\right\}$. If $\alpha_{r} \in \Psi$ then $-\alpha_{r} \in r(\Psi) \cap r\left(\Phi^{+}\right)$and so $r \in\left\{t_{\alpha} \mid \alpha \in r(\Psi) \cap\right.$ $\left.r\left(\Phi^{+}\right)\right\}$. This implies that $r \cdot A_{\Psi}=\left\{t_{\alpha} \mid \alpha \in r(\Psi) \cap \Phi^{+}\right\}$. If $\alpha_{r} \notin \Psi$ then $r \notin\left\{t_{\alpha} \mid \alpha \in\right.$ $\left.r(\Psi) \cap r\left(\Phi^{+}\right)\right\}$. But $\alpha_{r} \in r(\Psi)$ so $\{r\}+\left\{t_{\alpha} \mid \alpha \in r(\Psi) \cap r\left(\Phi^{+}\right)\right\}=\left\{t_{\alpha} \mid \alpha \in r(\Psi) \cap\right.$ $\left.\Phi^{+}\right\}$. Thus, in both cases $r \cdot A_{\Psi}=\left\{t_{\alpha} \mid \alpha \in r(\Psi) \cap \Phi^{+}\right\}$.

Since we are considering $(W, S)$ dihedral, we choose an orientation of the plane spanned by $\Phi$. Then for any root $\alpha \in \Phi$ we can define the neighbor of $\alpha$, denoted $n b r(\alpha)$, to be the root lying directly next to $\alpha$ if we traverse the root system clockwise. Recall that $S=\{r, s\}$. Interchanging $r$ and $s$ if necessary, we assume without loss of generality that $\alpha_{r}=n b r\left(-\alpha_{s}\right)$. We note that this implies that $n b r\left(\alpha_{s}\right) \notin \Phi^{+}$.

Lemma 3.2 Let $(W, S)$ be dihedral. Suppose $\alpha \in \Phi^{+}$and $\gamma:=n b r(\alpha) \in \Phi^{+}$. Then $t_{\alpha} t_{\gamma}=s r$.

Proof Let $(r s r \ldots)_{n}$ denote an element with $n$ simple reflections listed (i.e. $\ell\left((r s r \ldots)_{n}\right)$ is not necessarily $n)$. With this terminology, $t \in T$ can be written $t=(r s r \ldots)_{2 i+1}$ or $t=(\operatorname{srs} \ldots)_{2 i+1}$ for some $i \geq 0$. Under the chosen orientation for $\Phi^{+}$, if $t_{\alpha}=(r s r \ldots)_{2 i+1}$ then we can write $t_{\gamma}=(r s r \ldots)_{2 i+3}$ so that $t_{\alpha} t_{\gamma}=$ $(r s r \ldots)_{2 i+1}(r s r \ldots)_{2 i+3}=s r$. Otherwise, if $t_{\alpha}=(\operatorname{srs} \ldots)_{2 i+1}(i \geq 1)$ then $t_{\gamma}=$ $(\operatorname{srs} \ldots)_{2 i-1}$ and so $t_{\alpha} t_{\gamma}=s r$.

Now, given $A=A \Psi$, we introduce two conditions that a positive system, $\Gamma^{+}$, of $\Phi$ can have:

C1: There are roots $\alpha, n b r(\alpha) \in \Gamma^{+}$such that $\alpha \notin \Psi$ and $n b r(\alpha) \in \Psi$

C2: There are roots $\beta, \operatorname{nbr}(\beta) \in \Gamma^{+}$such that $\beta \in \Psi$ with $\operatorname{nbr}(\beta) \notin \Psi$

Lemma 3.3 Let $(W, S)$ be dihedral and let $A=A_{\Psi} \subseteq T$.

1. If the positive system $r\left(\Phi^{+}\right)$has condition $\mathbf{C 1}$, then there exists a path $1 \rightarrow x \rightarrow s r$ in $\Omega_{(W, A)}$.

2. If the positive system $r\left(\Phi^{+}\right)$has condition $\mathbf{C 2}$, then there exists a path $\mathrm{s} r \rightarrow x \rightarrow 1$ in $\Omega_{(W, A)}$.

Proof Replacing $A$ by $A+T$ reverses the orientation of edges in $\Omega_{(W, A)}$ and so 2 follows from 1 .

We now prove 1. There are two cases to consider. If $\alpha \neq \alpha_{s}$, then both $\alpha$ and $\gamma:=n b r(\alpha) \in \Phi^{+}$. So $t_{\alpha} \notin A$ and $t_{\gamma} \in A$. Also, since $t_{\alpha} \notin\{r, s\}$, it follows that $t_{\gamma} \in$ $N\left(t_{\alpha}\right)$. So we have that $t_{\gamma} \notin N\left(t_{\alpha}\right)+A$. Thus, we have a path $1 \rightarrow t_{\alpha} \rightarrow t_{\alpha} t_{\gamma}=s r$, 
where the last equality follows from Lemma 3.2. Now, if $\alpha=\alpha_{s}$ then $n b r(\alpha)=-\alpha_{r}$. Since $-\alpha_{r} \in \Psi$ we know that $\alpha_{r} \notin \Psi$ which implies $r \notin A$. Also, $s \notin A$ and clearly $r \notin N(s)$. Together, we see that there is a path $1 \rightarrow s \rightarrow s r$ in $\Omega_{(W, A)}$.

Lemma 3.4 For $(W, S)$ dihedral, let $A=A_{\Psi} \subseteq T$. If there are no 4-cycles in $\Omega_{(W, A)}$ then there is no positive system, $\Gamma^{+} \subset \Phi$ satisfying $\mathbf{C 1}$ and $\mathbf{C 2}$.

Proof Suppose there is a positive system $\Gamma^{+}$satisfying $\mathbf{C 1}$ and $\mathbf{C 2}$. It is clear that $-\Gamma^{+}$also satisfies $\mathbf{C 1}$ and $\mathbf{C 2}$. Since $\Gamma^{+}$satisfies both $\mathbf{C 1}$ and $\mathbf{C 2}$, then $w\left(\Gamma^{+}\right)$ will also satisfy both conditions ( $w$ with even length respects both conditions and $w$ with odd length interchanges the conditions). Thus, we can find a $w \in W$ such that $r\left(\Phi^{+}\right)=w\left(\Gamma^{+}\right)$or $r\left(\Phi^{+}\right)=w\left(-\Gamma^{+}\right)$. So we have that $r\left(\Phi^{+}\right)$satisfies $\mathbf{C 1}$ and $\mathbf{C 2}$ with respect to $A_{w(\Psi)}=w \cdot A_{\Psi}$. Since $\Omega_{(W, A)}$ is isomorphic to $\Omega_{(W, w \cdot A)}$, we can assume without loss of generality that $r\left(\Phi^{+}\right)$satisfies $\mathbf{C 1}$ and $\mathbf{C 2}$ with respect to $A_{\Psi}$. Thus, Lemma 3.3 implies that we have a path $s r \rightarrow u \rightarrow 1 \rightarrow v \rightarrow s r$ in $\Omega_{(W, A)}$.

Lemma 3.5 Let $(W, S)$ be dihedral and $A=A_{\Psi} \subseteq T$. If there is no positive system, $\Gamma^{+}$, of $\Phi$ satisfying $\mathbf{C 1}$ and $\mathbf{C 2}$, then $A \in \mathbf{A}_{(W, S)}$.

Proof Let $A \notin \mathbf{A}_{(W, S)}$. Recall that the only two reflection orders on $(W, S)$ are $\prec$ and $\prec^{\prime}$ described in Definition 2.1. Since $A$ is not an initial section of either of these orders, without loss of generality (replacing $A$ with $T \backslash A$ if necessary) we can find $t_{0} \in A$ and $t_{1}, t_{2} \in T \backslash A$ such that $t_{1} \prec t_{0}$ and $t_{2} \prec^{\prime} t_{0}$, i.e. $t_{2} \prec t_{0} \prec t_{1}$.

Now, if $(W, S)$ is finite, we can list all of the reflections and thus can find $t^{\prime}, t^{\prime \prime} \in T$ such that $t_{2} \preceq t^{\prime} \prec t_{0} \preceq t^{\prime \prime} \prec t_{1}$ with $\alpha_{t^{\prime}} \notin \Psi$ and $n b r\left(\alpha_{t^{\prime}}\right) \in \Psi$, and $\alpha_{t^{\prime \prime}} \in \Psi$ and $n b r\left(\alpha_{t^{\prime \prime}}\right) \notin \Psi$. Thus $\Phi^{+}$satisfies $\mathbf{C 1}$ and $\mathbf{C 2}$.

If ( $W, S)$ is infinite, then $T=T_{r} \cup T_{S}$ (disjoint union) where $T_{r}=\{t \in T \mid r \in$ $N(t)\}$ and $T_{s}=\{t \in T \mid s \in N(t)\}$. Suppose $\Phi^{+}$does not satisfy C1 and C2. We cannot have $t_{1}, t_{2}, t_{0} \in T_{u}$ for some $u \in\{r, s\}$ since this would imply, by the reasoning for $(W, S)$ finite, that $\Phi^{+}$satisfies $\mathbf{C 1}$ and $\mathbf{C 2}$. So, we can assume that $t_{2}, t_{0} \in T_{r}$ and $t_{1} \in T_{S}$ (the case $t_{2} \in T_{r}$ and $t_{0}, t_{1} \in T_{S}$ is exactly similar). Additionally, since $\mathbf{C 1}$ and C2 aren't both satisfied by $\Phi^{+}$, we may assume the following conditions hold (see Figure 1 above):

1. $\alpha_{t_{0}}=n b r\left(\alpha_{t_{2}}\right)$,

2. $t \in T \backslash A$ if $t \preceq t_{2}$,

3. $t \in T \backslash A$ if $t \in T_{s}$ and $t \preceq t_{1}$,

4. $t \in A$ if $t \in T_{r}$ and $t_{0} \preceq t$,

5. $t \in A$ if $t_{1} \prec t$.

Now, consider the positive system $\Gamma^{+}$with simple roots $\alpha_{t_{0}}$ and $-n b r\left(\alpha_{t_{0}}\right)$. Then $\Gamma^{+}$satisfies $\mathbf{C 1}$ using the roots $\alpha_{t_{2}}$ and $\alpha_{t_{0}}=n b r\left(\alpha_{t_{2}}\right)$. Also, by above, $\alpha_{t_{1}} \notin \Psi$ and $n b r\left(\alpha_{t_{1}}\right) \in \Psi$ (note that even if $t_{1}=s$ this is true since $n b r\left(\alpha_{s}\right)=-\alpha_{r} \in \Psi$ because by above $\left.\alpha_{r} \notin \Psi\right)$. Using this, we see that $\Gamma^{+}$satisfies $\mathbf{C 2}$ using the roots $-\alpha_{t_{1}}$ and $n b r\left(-\alpha_{t_{1}}\right)=-n b r\left(\alpha_{t_{1}}\right)$ (again see Figure 1).

With the dihedral case taken care of, we proceed to the general case. For the remainder of the paper, we assume that $(W, S)$ is a general Coxeter system. 
Fig. 1 This is a schematic diagram of the root system for $(W, S)$ infinite. $\Phi^{+}$consists of the roots which are non-negative linear combinations of $\alpha_{r}$ and $\alpha_{s}$. The dotted ray through the origin represents a limit line of roots. If $\mathbf{C 1}$ and $\mathbf{C 2}$ are not both satisfied by $\Phi^{+}$then $\Psi$ is pictured above with the roots in $\Psi$ labeled by $\bullet$, and those not in $\Psi$ labeled by $\circ$

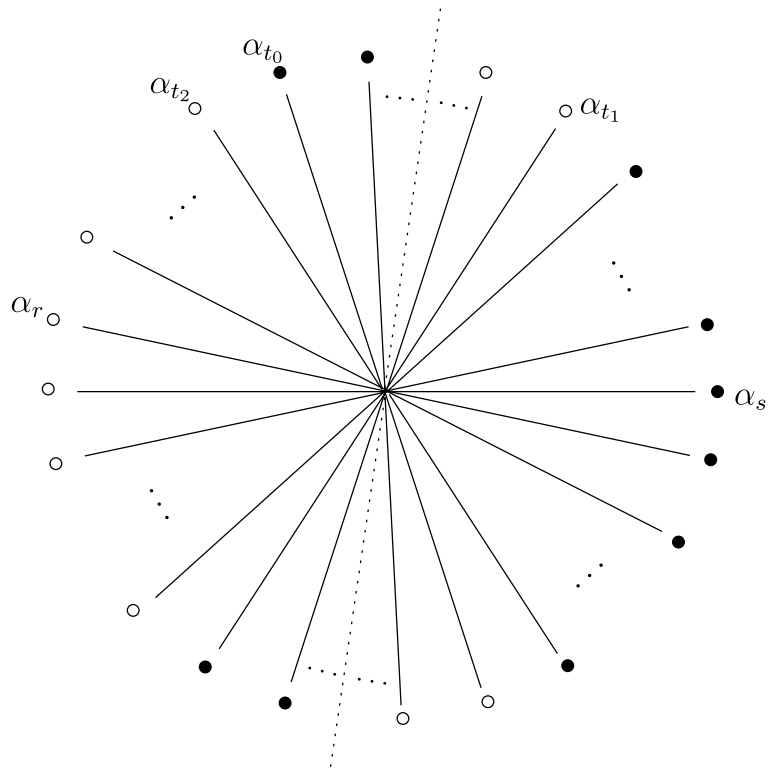

Proposition 3.6 For all $w \in W$ and $A \in \hat{\mathbf{A}}_{(W, S)}, w \cdot A \in \hat{\mathbf{A}}_{(W, S)}$.

Proof It suffices to check the condition for $w=r \in S$. Let $W^{\prime}$ be a dihedral reflection subgroup. If $r \in W^{\prime}$, then $(r \cdot A) \cap W^{\prime}=N_{\left(W^{\prime}, \chi\left(W^{\prime}\right)\right)}(r)+r\left(A \cap W^{\prime}\right) r \in \mathbf{A}_{\left(W^{\prime}, \chi\left(W^{\prime}\right)\right)}$ by [4]. Now, if $r \notin W^{\prime}$, then $(r \cdot A) \cap W^{\prime}=r\left(A \cap r W^{\prime} r\right) r$. However, conjugation by $r$ defines an isomorphism $\left(W^{\prime}, \chi\left(W^{\prime}\right)\right) \cong\left(r W^{\prime} r, r \chi\left(W^{\prime}\right) r\right)$ in this case, and by assumption $A \cap r W^{\prime} r \in \mathbf{A}_{\left(r W^{\prime} r, r \chi\left(W^{\prime}\right) r\right)}$, thus $(r \cdot A) \cap W^{\prime} \in \mathbf{A}_{\left(W^{\prime}, \chi\left(W^{\prime}\right)\right)}$.

Proposition 3.7 Let $A \in \hat{\mathbf{A}}_{(W, S)}, x \in W, t \in T$. Then $\ell_{A}(x, x t)>0$ iff $t \notin N\left(x^{-1}\right)+A$.

Proof Because of Proposition 3.6, the argument will follow directly from the proof of [3] (Proposition 1.2) which only requires that $A \in \hat{\mathbf{A}}_{(W, S)}$.

We now can turn to the proof of Theorem 2.3:

Proof $(1) \Leftrightarrow(2) \Rightarrow(3)$ is clear.

(3) $\Rightarrow(4)$ : By [2] (Lemma 3.2), we know that for a dihedral subgroup $W^{\prime}$ of $W$, $N_{\left(W^{\prime}, S^{\prime}\right)}(w)=N_{(W, S)}(w) \cap W^{\prime}$. This implies $\Omega_{\left(W^{\prime}, A \cap W^{\prime}\right)}$ is a subgraph of $\Omega_{(W, A)}$. So, if $A \notin \hat{\mathbf{A}}_{(W, S)}$, Lemma 3.4 and Lemma 3.5 imply that there exists some dihedral subgroup $W^{\prime}$ of $W$ with $\Omega_{\left(W^{\prime}, A \cap W^{\prime}\right)}$ containing a cycle with four edges.

(4) $\Rightarrow(5)$ : Suppose that $A \in \hat{\mathbf{A}}_{(W, S)}, x \in W$ and $t \in N\left(x^{-1}\right)+A$. Then Proposition 3.7 implies that $\ell_{A}(x, x t)<0$. But by [3] $\ell_{A}(1, x)+\ell_{A}(x, x t)=\ell_{A}(1, x t)$ and this implies $\ell_{A}(x t)-\ell_{A}(x)=\ell_{A}(x, x t)<0$.

$(5) \Rightarrow(1)$ : Suppose $\Omega_{(W, A)}$ has a cycle. This means that $x t_{1} \ldots t_{n}=x$ for some $n>0$ (all $t_{i} \in T$ and with $t_{i} \notin N\left(\left(x t_{1} \ldots t_{i-1}\right)^{-1}\right)+A$ for all $\left.i\right)$. By assumption, $\ell_{A}(x)<$ $\ell_{A}\left(x t_{1}\right)<\ell_{A}\left(x t_{1} t_{2}\right)<\ldots<\ell_{A}\left(x t_{1} t_{2} \ldots t_{n}\right)=\ell_{A}(x)$ which is a contradiction. 
Remark 3.8 In case $(W, S)$ is finite, say of order $m$, we can give a much simpler proof of the equivalence of (1), (2), (4), and (5). By the proof above, we have (4) $\Rightarrow(5) \Rightarrow(1) \Leftrightarrow(2)$. If (4) fails, then $w \cdot A \neq T$ for all $w \in W$ (or else $A=w^{-1} \cdot T$ which is an initial section). So we can choose $t \notin w \cdot A$. It follows that we can recursively choose $t_{1}, \ldots, t_{m}$ such that $t_{1} \notin A$ and $t_{i} \notin t_{i-1} \cdots t_{1} \cdot A$ for $i=2, \ldots, m$. This gives us the following path in $\Omega_{(W, A)}: 1 \rightarrow t_{1} \rightarrow \ldots \rightarrow t_{m} \cdots t_{1}$. However, this path has $m+1$ elements and $W$ has $m$ elements, so there must be two elements in the path that are the same thus creating a cycle.

\section{References}

1. Björner, A., Brenti, F.: Combinatorics of Coxeter Groups. Graduate Texts in Mathematics, vol. 231. Springer, New York (2005) MR2133266

2. Dyer, M.: Reflection subgroups of Coxeter systems. J. Algebra 135(1), 57-73 (1990) MR1076077 $(91 \mathrm{j}: 20100)$

3. Dyer, M.J.: Hecke Algebras and Shellings of Bruhat Intervals. II. Twisted Bruhat Orders, KazhdanLusztig Theory and Related Topics, Chicago, 1989. Contemp. Math., vol. 139, pp. 141-165. Am. Math. Soc., Providence (1992) MR1197833 (94c:20072)

4. Dyer, M.J.: Hecke algebras and shellings of Bruhat intervals. Compos. Math. 89(1), 91-115 (1993) MR1248893 (95c:20053)

5. Humphreys, J.E.: Reflection Groups and Coxeter Groups. Cambridge Studies in Advanced Mathematics, vol. 29. Cambridge University Press, Cambridge (1990) MR1066460 (92h:20002) 